\title{
Motion and Positional Error Correction for Cone Beam 3D-Reconstruction with Mobile C-Arms
}

\author{
C. Bodensteiner ${ }^{1}$, C. Darolti ${ }^{2}$, H. Schumacher ${ }^{3}$, L. Matthäus ${ }^{1}$, \\ and A. Schweikard ${ }^{1}$ \\ ${ }^{1}$ Institute of Robotics and Cognitive Systems, University of Lübeck, Ger \\ ${ }^{2}$ Institute for Signal Processing, University of Lübeck, Ger \\ ${ }^{3}$ Institute of Mathematics, University of Lübeck, Ger \\ bodensteiner@rob.uni-luebeck.de.
}

\begin{abstract}
CT-images acquired by mobile C-arm devices can contain artefacts caused by positioning errors. We propose a data driven method based on iterative 3D-reconstruction and 2D/3D-registration to correct projection data inconsistencies. With a $2 \mathrm{D} / 3 \mathrm{D}$-registration algorithm, transformations are computed to align the acquired projection images to a previously reconstructed volume. In an iterative procedure, the reconstruction algorithm uses the results of the registration step. This algorithm also reduces small motion artefacts within 3D-reconstructions. Experiments with simulated projections from real patient data show the feasibility of the proposed method. In addition, experiments with real projection data acquired with an experimental robotised C-arm device have been performed with promising results.
\end{abstract}

\section{Introduction}

Modern mobile C-arm devices allow for intraoperative 3D-reconstruction. Unfortunately, compared to conventional (fixed) CT-scanners, 3D-reconstructions from these devices show greater artefacts due to hardware tradeoffs required for mobile usage. Inconsistencies caused by noise, mechanical flex, geometric distortions, patient motion and errors in positioning the $\mathrm{C}$-arm affect the 3Dreconstruction results. In this paper we present a method to compensate for positioning error inconsistencies in projection data. This approach is also capable of eliminating small motion artefacts within 3D-reconstructions by modelling them as a virtual x-ray camera repositioning.

Forward projection registration algorithms have been successfully used to compensate for motion in SPECT imaging [1. Inspired by this work, we now combine a conventional rigid 2D/3D-registration algorithm with an iterative reconstruction algorithm. To our best knowledge, this approach has not been used for cone beam 3D-reconstruction before.

We use the averaging nature of algebraic 3D-reconstruction techniques to correct the position and orientation of the acquired projections by means of registration. In the 2D/3D-registration step, 3D-transformations of the positions of the $\mathrm{x}$-ray cameras are calculated to minimize a distance measure between the 
acquired projections and a set of forward projections. The forward projections are digital reconstructed radiographs (DRR) generated from the previously reconstructed volume through virtual x-ray cameras at new positions. As a result, an iterative reconstruction scheme arises which is robust with respect to small positional error and rigid motion artefacts.

\section{Method}

Cone beam 3D-reconstruction with algebraic methods can be formulated as the following linear least-squares problem: find $f \in \mathbf{R}_{+}^{n}$ such that

$$
R(f)=\|A f-b\|=\min !
$$

where $A$ denotes a projection-operator (also called system matrix) simulating the cone beam projections, $f$ the reconstruction volume, $n$ the image size, and $b$ the measured projections. In a first step only positional errors of the C-arm device are considered. Assuming that x-ray camera transformations can be modeled as $3 \mathrm{D}$ rigid object motion transformations, this leads to the following minimization problem:

$$
J\left(\begin{array}{c}
f \\
\Phi
\end{array}\right)=\sum_{i=1}^{M}\left\|A_{i} f\left(\Phi_{i}\right)-b_{i}\right\|=\min !
$$

Here $A_{i}$ denotes the $\mathrm{i}$-th part of the projection operator simulating one cone beam projection image $b_{i}, \mathrm{M}$ the number of acquired projection images and $\Phi_{i}$ the $3 \mathrm{D}$ rigid motion transformation of the reconstructed object with six degrees of freedom. An equation similiar to Eqn.(2) is minimized in an iterative procedure to compensate for motion in SPECT imaging 2. Our approach is to first solve Eqn.(11) with a reconstruction algorithm. In a second step the motion parameters of Eqn.(2) are estimated with a rigid 2D/3D-registration algorithm by employing an appropriate distance measure [3]. The motion parameters are then used to correct the x-ray camera positions. In the next iteration, the reconstruction algorithm uses the information from the corrected projections to compute a new least-squares solution with respect to all projection equations given in Eqn.(1). This procedure is then iteratively repeated until some convergence criteria are fulfilled. The corrected projection positions obtained with this method represent an equal or better projection geometry with respect to the used distance measure, since we initialize the registration algorithm with the projection positions given by the C-arm device. For reasons of clarity and completeness a short description of the employed reconstruction algorithm and details of the $2 \mathrm{D} / 3 \mathrm{D}$-registration algorithm are given.

\subsection{D-Reconstruction Algorithm}

The algebraic reconstruction technique (ART) was used for 3D-reconstruction. Let $g_{i}(\lambda ; b ; \cdot), G(\lambda ; b ; \cdot): \mathbf{R}^{n} \rightarrow \mathbf{R}^{n}$ be the applications of:

$$
g_{i}(\lambda ; b ; f)=f-\lambda \frac{\left\langle f, a_{i}\right\rangle-b_{i}}{\left\|a_{i}\right\|^{2}} a_{i}, i=1, \cdots, m,
$$




$$
G(\lambda ; b ; f)=\left(g_{1} \circ \cdots \circ g_{m}\right)(\lambda ; b ; x),
$$

where $a_{i}$ denotes the i-th row of the system matrix $A$ and $b_{i}$ the i-th element of the measured projection image. Letting $\lambda$ denote a relaxation parameter, the algorithm of Kaczmarz calculates a limit point for the following iteration equation:

$$
f^{0} \in \mathbf{R}^{n} ; \quad k=0,1,2, \ldots ; \quad f^{k+1}=G\left(\lambda ; b ; f^{k}\right) .
$$

Censor et. al 4 . proved that for sufficiently small values of $\lambda$ the algorithm converges to a least squares solution of a weighted version of Eqn.(1). This technique has the advantage that it is able to employ a former reconstruction result in order to compute a new least squares solution. For further details and enhancements regarding the reconstruction of inconsistent projection data we refer to [5]

\subsection{D/3D-Registration}

2D/3D-registration algorithms are conventionally used to find the transformation between intraoperative $2 \mathrm{D}$ radiographs and a preoperative $\mathrm{CT}$-volume. In our application we have two significant advantages over conventional 2D/3Dregistration scenarios, since the imaged object does not change and we have a good initialization of the position in case of positional error correction. The main building blocks of these algorithms are the distance measure and the optimization scheme [3]. We only consider intensity based distance measures which do not require prior segmentation. We tested the sum of squared distances (SSD) for minimizing the positional inconsistencies. We also tested a gradient-based correlation distance measure [3]. In this case, the cost function to be optimized $C=\frac{1}{2}\left(C_{x}+C_{y}\right)$ is calculated as the average of the normalized cross correlations of the partial derivatives of the projection and DRR image intensities. $\operatorname{Pr}_{x}(x, y)=\frac{\partial \operatorname{Pr}(x, y)}{\partial x}$ and $\operatorname{Pr}_{y}(x, y)=\frac{\partial \operatorname{Pr}(x, y)}{\partial y}$ denote the partial derivatives of image intensities, and $\overline{P r_{x}}$ and $\overline{P r_{y}}$ denote their mean values.

$$
C_{x}=\frac{\sum_{x, y}\left(\operatorname{Pr}_{x}(x, y)-\overline{P r_{x}}\right)\left(D r_{x}(x, y)-\overline{D r r_{x}}\right)}{\sqrt{\sum_{x, y}\left(\operatorname{Pr}_{x}(x, y)-\overline{P r_{x}}\right)^{2}} \sqrt{\sum_{x, y}\left(D r r_{x}(x, y)-\overline{D r r_{x}}\right)^{2}}}
$$

The gradient cost function seems to be more robust with respect to positional inconsistencies in the data. In addition, problems caused by different image generation processes of DRRs and real projections are resolved as well.

The optimization was carried out in a hierarchical multi-level approach: a pyramid of images is constructed by down-sampling and filtering the image at multiples of 2 and the optimization is run for each level of the pyramid. This allows for a robust and fast optimization, since evaluating the cost function at lower levels is computationally very cheap.

The values of the cost functions for transformation parameters varying around the optimum found by the 2D/3D-registration algorithm have been plotted in Fig. 1. They all show a relatively smooth, monotonically increasing function with a unique maximum and an appropriate convergence range in the vicinity 
of the found value. Also, one can see that out-of-plane parameters (here the xdeviation) usually cannot be determined as accurately as other parameters due to the projection geometry [6]. A variant of the Hooke-Jeeves pattern-search 7 was used as an optimization-scheme. To avoid double function evaluations the function values were cached in a hash table as described in 8 .
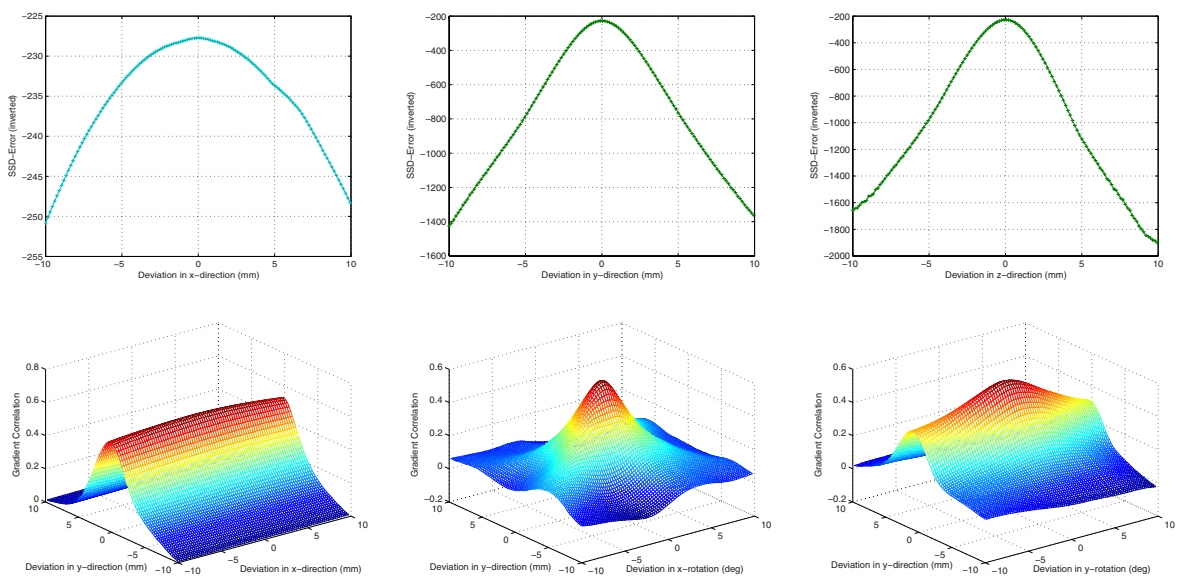

Fig. 1. Cost function values for the proximal femur experiment as a function of deviation from the optimal value found by the $2 \mathrm{D} / 3 \mathrm{D}$-registration algorithm. The upper row shows the values of SSD distance measure for deviations of the translational parameters $(x, y, z)$. The lower row shows the values of gradient cost function for deviations of two transformation parameters: $(x, y),\left(y, \theta_{x}\right),\left(y, \theta_{y}\right)$.

\subsection{Implementation Details and Computation Time}

All algorithms were implemented in $\mathrm{C}++$ and parallelized with OpenMP 9 . When using several processor cores, a almost linear speedup has been observed since all employed algorithms are inherently parallel. The registration of one projection image (Fig. 3, 568 2 pixel) took around $30 \mathrm{~s}-40$ s for a $256^{3}$ volume and 3 image pyramid levels. For fast DRR generation a shear warp factorization algorithm [6] was used. A typical complete registration iteration on our test system (Dual Xeon 5160, 16 GB Ram) took around 780s for a $256^{3}$ dataset and 100 projections $\left(568^{2}\right)$. One iteration of our software based reconstruction algorithm (double precision) took around 180s. To allow for intraoperative usage, a clear reduction of computation time must be obtained. Since DRR generation is the most time consuming part of the registration algorithm, our future implementation will make use of GPU based algorithms (e.g. 3D texture based DRR generation, GPU based reconstruction algorithms).

The entries of the system matrix A were determined by discretizing the ray beam with a trilinear interpolation coefficient approach [10]. The reconstruction volume was also optimized by a block structure such as to speed up memory access in order to enable a fast set up of the system matrix equations [10]. 


\subsection{Clinical- and C-Arm Projection Data Experiments}

We generated DRR's (100 projections, 568x568, 180 degree angle range) from a clinical dataset $(512 \times 512 \times 200)$ of a distal femur bone. After resampling the dataset to an isotrope $256^{3}$-grid, we perturbed all camera positions $T_{W \text { orld }}^{\text {Cam }}$ to $T_{\text {World }}^{\text {pertCam }}$ ( $\max \pm 10 \mathrm{~mm}, \max \pm 1 \mathrm{deg}$ ). The perturbed projections were generated from a different DRR generator as the one employed in the registration process in order to simulate the different image generation process. In a first experiment we measured the registration accuracy by registering 400 perturbed DRRs to the original volume. The euler angle representation of the deviation matrix Tm was used for evaluation.

$$
T m_{\text {regCam }}^{\text {Cam }}=T_{\text {regCam }}^{\text {World }} T_{W o r l d}^{\text {Cam }}
$$

We applied the iterative correction process to the perturbed data and in each step, we ran 5 ART-iterations to reconstruct the object. The projections where then iteratively registered to the previous reconstruction result.

The real projection data was acquired with a robotized experimental C-arm 11 . from Ziehm-Imaging (Fig. 2). The position of the C-arm is known from the direct kinematics. In addition this C-arm can be arbitrarily positioned to a point in its working space by computing the inverse kinematics solution described in [12].

In the first experiment a sawbone model of the proximal femur was imaged in a planar angular orbit (50 projections, 568x568, 180 degree angle range). A sequential ART algorithm with a small relaxation parameter $(\lambda=0.25)$ was used for reconstruction. After 6 ART-iterations per step, the original projection images were registered to the reconstruction result, and subsequently a new reconstruction was started.


Fig. 2. Left: the robotized C-arm prototype. Middle: determination of the geometrical distortions using bi-variate polynomials. Right: projection image of the rat skull lying in a cup with calibration grid.

In a second experiment we imaged a rat skull (60 projections, 568x568, 135 degree angle range). In this experiment, the geometric distortion was corrected using bi-variate polynomials of degree five [8]. The coefficients were determined 
using a calibration object placed on the image intensifier (Fig. 2). This step becomes obsolete for the next version of the robotized $\mathrm{C}$-arm device which will be equipped with digital flat panel detector technology, where no geometrical distortions occur.

\section{$3 \quad$ Results}

The registration accuracy of the clinical data experiment showed medium rotational deviations of $0.137(\mathrm{x}), 0.0134(\mathrm{y})$ and $0.07(\mathrm{z})$ degree and medium translational deviations of $0.05(\mathrm{x}), 0.70(\mathrm{y}), 0.35(\mathrm{z}) \mathrm{mm}$. In addition the visualization of the 2D/3D-registration results showed a clear positional error reduction (Fig. 33). In order to evaluate the correction algorithm results, we used the reconstruction residual (1), which is a direct measure for inconsistencies in the projection data. For the clinical dataset (Fig. 6) the residual dropped from initially 17019 to 7421 after 10 complete iterations (original data 2375).
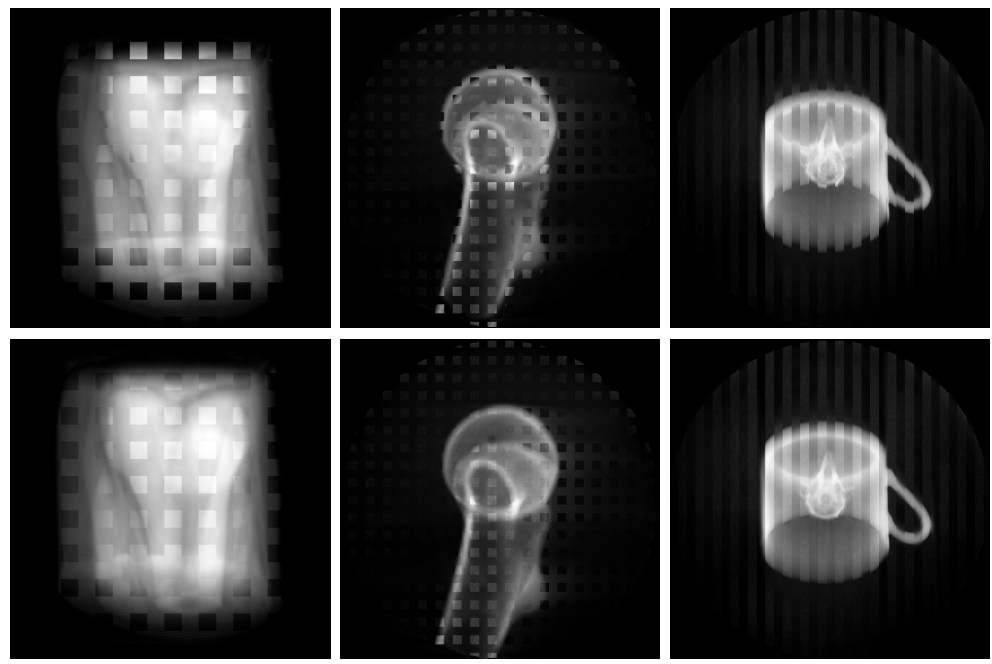

Fig. 3. Registration of one projection image with its DRR (top row unregistered) of the clinical distal femur bone (left), the sawbone proximal femur bone (middle) and the rat skull lying in a small cup (right)

Fig. 5 shows the reconstruction residual of the proximal femur experiment. The residual declines after each reconstruction-registration step. This gives evidence that the calculated positions lead to a more consistent reconstruction and in succession to a more accurate registration. In the rat skull experiment, a fast decline of the residiual could be observed, due to the prior removal of geometric distortions. Also a siginficant improvement in reconstruction quality could be observed after one iteration (Fig. 4) of the proposed correction scheme. 

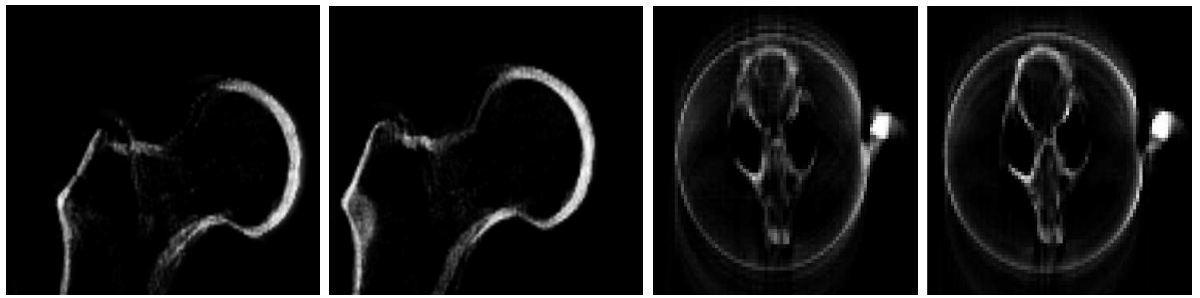

Fig. 4. Reconstructed slices from a proximal femur sawbone model and a rat skull. The respective left slice shows the uncorrected reconstruction result. Artefacts appearing as doubled, blurred edges and holes are significantly reduced by the iterative correction process (sawbone 7 iterations, rat skull 1 iteration).

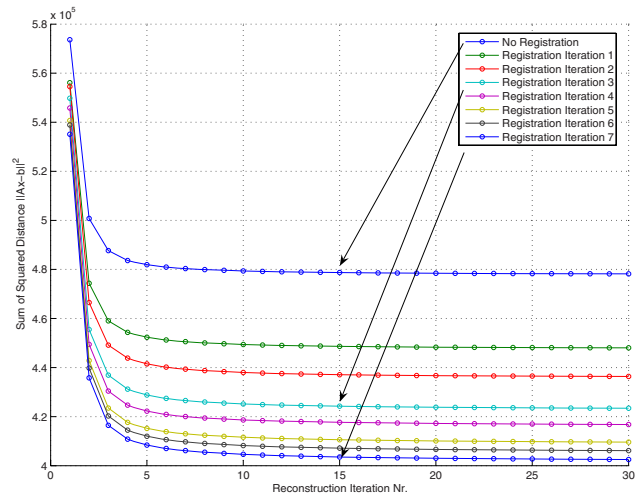

Fig. 5. Plot of the reconstruction residual (proximal femur experiment) in dependency of the reconstruction and registration iterations
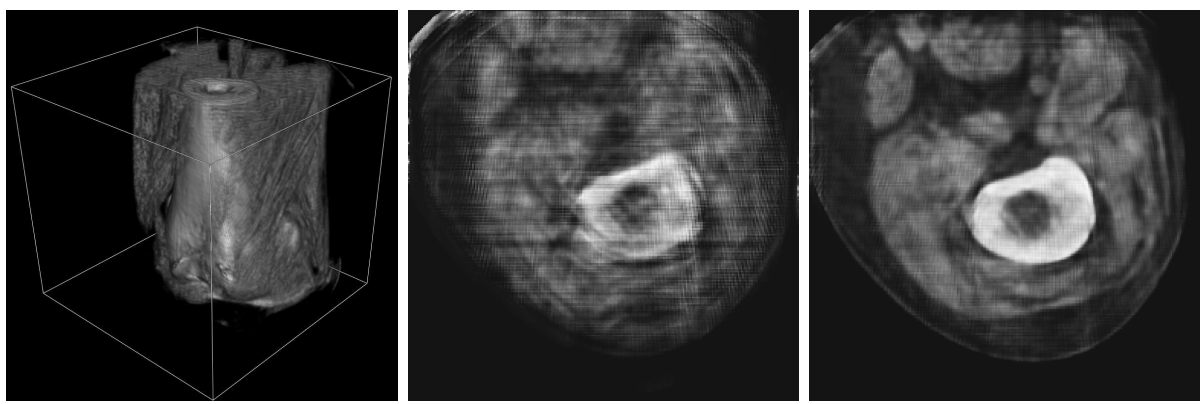

Fig. 6. Results from the clinical dataset of the distal femur. Reconstruction from original data, the volume rendering is clipped and visualized with a modified opacity function (left). Axial slice from the initial reconstruction from perturbed data (middle). Same slice after 10 iterations of the proposed algorithm (right). 


\section{Discussion}

In this paper we have implemented a new method for positional error and motion compensation by integrating a 2D/3D-registration algorithm into a cone beam 3D-reconstruction framework. In all experiments, the inconsistencies were clearly reduced resulting in significantly improved reconstructions. In the case of simulated projections, perturbing the projection data has the effect that the position and orientation of the original object is also slightly modified. This disturbes a simple comparison. We plan to perform a rigid registration of the two volumes to allow for a direct comparison of the reconstruction results. The effect of slight positional changes of the reconstructed object due to the correction process can be also an issue for intraoperative navigation applications, since the reconstruction result is sometimes directly used for registration free navigation. To allow an intraoperative usage, the registration and reconstruction time must be in the range of two minutes. This is achievable by using GPU based algorithms and modern parallel hardware. Another important issue is registration accuracy. When performing position correction the use of subsequent projection image subsets should improve registration accuracy and robustness. Also slight changes of intrinsic x-ray camera parameters should be taken into account to model the twisting and bending of the C-arm.

\section{References}

1. Lee, K.J., et al.: Use of forward projection to correct patient motion during SPECT imaging. Physics in Medicine and Biology 43, 171-187 (1998)

2. Schumacher, H., Fischer, B.: A new approach for motion correction in SPECT imaging. In: Bildverarbeitung fuer die Medizin (2007)

3. Penney, G., Weese, J., Little, J.A., et al.: A comparison of similarity measures for use in $2 \mathrm{~d}-3 \mathrm{~d}$ medical image registration. In: Medical Image Computing and Computer-Assisted Intervention p. 1153 (1998)

4. Censor, Y., Gordon, D., Gordon, R.: Strong underrelaxation in kaczmarz's method for inconsistent systems. Numer. Math. 41, 83-92 (1983)

5. Popa, C., Zdunek, R.: Kaczmarz extended algorithm for tomographic image reconstruction from limited-data. Mathematics and Computers in Simulation 65, 579-598 (2004)

6. Weese, J., Goecke, R., Penney, G., Desmedt, P., Buzug, T., Schumann, H.: Fast voxel-based $2 \mathrm{~d} / 3 \mathrm{~d}$ registration algorithm using a rendering method based on the shear-warp factorization. In: Proceedings of SPIE, pp. 802-810 (1999)

7. Parker, D.: An empirical investigation of the global behavior of several pattern search algorithms. In: Technical Report, Department of Computer Science - University of North Carolina (1999)

8. Dotter, M.: Flouroskopiebasierte Navigation zur intraoperativen Unterstuetzung orthopaedischer Eingriffe. PhD thesis, Technische Universitaet Muenchen (2006)

9. Chandra, R., Menon, R., Daqum, L., et al.: Parallel Programming in OpenMP. Morgan Kaufmann, San Francisco (2001) 
10. Mueller, K.: Fast and accurate three-dimensional reconstruction from cone-beam projection data using algebraic methods. PhD thesis, Ohio State University (1998)

11. Binder, N., Bodensteiner, C., Matthaeus, L., et al.: Image guided positioning for an interactive c-arm fluoroscope. In: Computer Assisted Radiology and Surgery (CARS) (2006)

12. Matthaeus, L., Binder, N., Bodensteiner, C., et al.: Closed form inverse kinematic solution for fluoroscopic c-arms. Advanced Robotics 21 (2007) 